\title{
Disturbance Observer Based Adaptive Sliding Mode Control for a Continuous Stirred Tank Reactor
}

\author{
Zhang Ye $^{1}$, ZHAO Dongya ${ }^{1}$, SPURGEON Sarah K. ${ }^{2,1}$ \\ 1. College of Chemical Engineering, China University of Petroleum, Qingdao 266555, P. R. China \\ E-mail: dyzhao@upc.edu.cn;dongyazhao@139.com \\ 2. Department of Electronic \& Electrical Engineering, University College London, Torrington Place, London WC1E 7JE, United Kingdom \\ E-mail: s.spurgeon@ucl.ac.uk
}

\begin{abstract}
The continuous stirred tank reactor (CSTR) typifies an important class of process control systems. Is is a nonlinear system and is sensitive to both external disturbances and system uncertainty. Given these challenges, a nonsingular terminal sliding mode observer is proposed to estimate any external disturbance. Then, a continuous adaptive sliding mode control method is combined with the proposed disturbance observer. This is found to reduce chattering and improve control accuracy when compared with other methods. A full Lyapunov stability proof of the resulting closed-loop system is performed and the effectiveness of the proposed approach is demonstrated by simulation experiments.
\end{abstract}

Key Words: CSTR, disturbance observer, adaptive sliding mode control

\section{Introduction}

The CSTR is a typical process control unit which is used for polymerization, condensation and other reaction processes in the chemical industry. The unit has the potential to increase the rate and adequacy of chemical reactions [1]. From the viewpoint of control, the dynamic model of a CSTR is nonlinear, and the system is subject to external disturbances and system uncertainty [2-5]. These characteristics provide control challenges and a number of methods have been proposed to deal with such complex systems, including neural network control [6], optimal output tracking control [7] and model predictive control [8]. Sliding mode control is another good candidate control system for the CSTR due to its excellent ability to deal with external disturbances and system uncertainty.

Sliding mode control has some advantages, such as, strong robustness, straightforward design, low computational complexity and ease of practical application [9-11]. However, for traditional first order sliding mode control, high gain control may be required to achieve a rapid rate of convergence and strong robustness properties. This can in turn lead to control input saturation as the initial control values required in particular may be large. In addition, the interaction of any nonsmooth term in the reaching law with non-ideal dynamics may induce chattering.

There are many methods to reduce chattering in the sliding mode control literature. A boundary layer technique is defined for nonlinear systems [12] to suppress chattering, but the finite time reaching properties are lost and the robustness is weakened. Second order and high order sliding control may be utilized to attenuate chattering and preserve robustness[13, 14]. However, the design process can be more complicated. A continuous terminal sliding mode control approach can achieve a reaching mode with reduced chattering [15], but high gain is required to accommodate system uncertainty and disturbances which may cause control input saturation.

This work is supported by National Natural Science Foundation (NNSF) of China under Grant 61473312
Another way to reduce the amplitude of chattering is to use a disturbance observer within the control strategy. A disturbance observer can be used to estimate uncertainty and disturbances online, and direct compensation can be used to ammeliorate the effects which in turn reduces chattering. For example, sliding mode disturbance observers are proposed based on super twisting algorithms in $[16,17]$. In order to simplify the strategies above and reduce chattering, a disturbance reconstruction approach is presented using the integral of sign function in [18]. A linear disturbance observer is employed in [19] to reduce the gain of the switching term. However, the aforementioned approaches do not consider the problems that may arise in implementation, such as the effects caused by overcompensation.

Overcompensation happens when the control gain is far larger than the variable uncertainty. In this case, an adaptive law can track the variation in the uncertainty and disturbance, which can reduce the conservatism and prevent overcompensation. New methodologies are proposed in [20] to obtain a robust sliding mode adaptive-gain control law. Further, a novel super-twisting adaptive sliding mode control law is presented to control an electropneumatic actuator [21]. The adaptation algorithm does not overestimate even in the case where the bounds on the uncertainty and disturbance are unknown.

In this paper, a novel disturbance observer is used to estimate the uncertainty and disturbance in finite time, and it ensures a rapid response. Moreover, an adaptive sliding mode control for the CSTR is designed according to [22]. The proposed approach seeks to reduce conservatism and avoid overcompensation as required to reduce the amplitude on any chattering effectively.

The paper is organized as follows: The dynamic equations of a CSTR are presented in Section 2. The disturbance observer is designed and the Lyapunov stability proof is given in Section 3. A continuous adaptive sliding mode control method is proposed based on the observer designed in Section 4. In Section 5, simulation experiments are presented to validate the effectiveness of the proposed approach. Concluding remarks are drawn in Section 6. 


\section{Problem Formulation}

The reaction in the CSTR is considered to be first order, exothermic and irreversible, and a dimensionless dynamic model from [23] is used as follows:

$$
\begin{aligned}
\dot{x}_{1}= & -a x_{1}+D a\left(1-x_{1}\right) \exp \left(\gamma x_{2} /\left(\gamma+x_{2}\right)\right) \\
\dot{x}_{2}= & -a x_{2}-b D a\left(1-x_{1}\right) \exp \left(\gamma x_{2} /\left(\gamma+x_{2}\right)\right) \\
& +\beta\left(u-x_{2}\right)+d \\
y= & x_{2}
\end{aligned}
$$

where $x_{1}, x_{2} \in R$ are the states, and represent the dimensionless concentration and temperature respectively. $y$ is the system output, and represents the dimensionless temperature. $d \in R$ is the external disturbance and the system uncertainty in the input channel. The details of the dimensionless parameters of (1) can be found in [23]. Let $f(x)=-a x_{2}-b D a\left(1-x_{1}\right) \exp \left(\gamma x_{2} /\left(\gamma+x_{2}\right)\right)-\beta x_{2}$, then (1) is written as follows:

$$
\begin{aligned}
& \dot{x}_{1}=-a x_{1}+D a\left(1-x_{1}\right) \exp \left(\gamma x_{2} /\left(\gamma+x_{2}\right)\right) \\
& \dot{x}_{2}=f(x)+\beta u+d \\
& y=x_{2}
\end{aligned}
$$

Assumption 1. There exists $\bar{d}>0$ satisfying $|\dot{d}| \leq \bar{d}$.

Assumption 2. The states of (2) are measurable.

Assuming the expected temperature signal of the CSTR system is $x_{2 d}$ based on Assumption 2, then the temperature error and its derivative are defined as

$$
\left\{\begin{array}{c}
e=x_{2}-x_{2 d} \\
\dot{e}=\dot{x}_{2}-\dot{x}_{2 d}
\end{array}\right.
$$

\section{Design of the Disturbance Observer}

External disturbances are always present in a CSTR system. Consequently, a disturbance observer is designed in this section to reduce the effect of the disturbance on the controller performance. Firstly, a symbol is defined as follows to simplify subsequent formulae:

$$
\operatorname{sig}(\rho)^{\gamma}=|\rho|^{\gamma} \operatorname{sgn}(\rho)
$$

A terminal sliding mode is presented as below:

$$
\begin{gathered}
\left\{\begin{array}{l}
s_{*}=c\left(x_{2}-\hat{x}_{2}\right) \\
\Delta(t)=-\alpha\left|x_{2}-\hat{x}_{2}\right|+\alpha\left|x_{2}(0)-\hat{x}_{2}(0)\right| \\
s_{0}=s_{*}-s_{*}(0) \exp (\Delta(t)) \\
\dot{\hat{x}}_{2}=f(x)+\beta u+\hat{d} \\
s_{1}=\dot{s}_{0}+\Gamma_{1} s_{0}+\Gamma_{2} P\left(s_{0}\right)
\end{array}\right. \\
P\left(s_{0}\right)=\left\{\begin{array}{l}
\left|s_{0}\right|^{\theta} \operatorname{sgn}\left(s_{0}\right),\left|s_{0}\right| \geq \varepsilon \\
(1-\delta) \varepsilon^{\theta} \sin \left(\pi /(2 \varepsilon) s_{0}\right) \\
+\delta \varepsilon^{\theta} \operatorname{sgn}\left(s_{0}\right),\left|s_{0}\right|<\varepsilon
\end{array}\right.
\end{gathered}
$$

where $c>0, \alpha>0, \Gamma_{1}>0, \Gamma_{2}>0, \theta=p / q, 0<p<q$, $p$ and $q$ are odd numbers, $\varepsilon>0,0<\delta<1, \hat{d}$ is an estimate of $d$.

Defining the corresponding disturbance estimation error as

$$
\tilde{d}=d-\hat{d}
$$

According to (5),

$$
\begin{aligned}
\dot{s}_{0}= & \dot{s}_{*}-s_{*}(0) \exp (\Delta(t)) \dot{\Delta}(t) \\
= & {\left[c+\alpha s_{*}(0) \exp (\Delta(t)) \operatorname{sgn}\left(x_{2}-\hat{x}_{2}\right)\right] } \\
& \left(\dot{x}_{2}-\dot{\hat{x}}_{2}\right)
\end{aligned}
$$

Defining $\kappa_{1}=c+\alpha s_{*}(0) \exp (\Delta(t)) \operatorname{sgn}\left(x_{2}-\hat{x}_{2}\right)$, then (8) can be written as

$$
\dot{s}_{0}=\kappa_{1} \tilde{d}
$$

Defining $\kappa_{2}=1 / \kappa_{1}$, an intermediate variable is available as

$$
\tilde{d}=\kappa_{2} \dot{s}_{0}
$$

Differentiating (9) yields

$$
\begin{aligned}
\ddot{s}_{0}= & \dot{\kappa}_{1} \tilde{d}+\kappa_{1} \dot{\tilde{d}} \\
= & -\alpha^{2} \mathrm{~s}_{*}(0) \exp (\Delta(t)) \operatorname{sgn}\left(x_{2}-\hat{x}_{2}\right) \\
& \operatorname{sgn}\left(x_{2}-\hat{x}_{2}\right) \tilde{d}^{2}+\kappa_{1} \dot{\tilde{d}}
\end{aligned}
$$

Substituting (10) into (11),

$$
\begin{aligned}
\ddot{s}_{0}= & -\alpha^{2} \mathrm{~S}_{*}(0) \exp (\Delta(t)) \operatorname{sgn}\left(x_{2}-\hat{x}_{2}\right) \\
& \operatorname{sgn}\left(x_{2}-\hat{x}_{2}\right) \dot{s}_{0}^{2} / \kappa_{1}{ }^{2}+\kappa_{1} \dot{\tilde{d}}
\end{aligned}
$$

Defining $\kappa_{3}=\exp (\Delta(t)) \operatorname{sgn}\left(x_{2}-\hat{x}_{2}\right) \operatorname{sgn}\left(x_{2}-\hat{x}_{2}\right)$ $\dot{s}_{0}^{2} / \kappa_{1}^{2}$, so (12) can be expressed as

$$
\ddot{s}_{0}=-\kappa_{3} \alpha^{2} \mathrm{~S}_{*}(0)+\kappa_{1} \dot{\tilde{d}}
$$

Substitution of (13) into (5) yields

$$
\begin{aligned}
\dot{s}_{1} & =\ddot{s}_{0}+\Gamma_{1} \dot{s}_{0}+\Gamma_{2} \dot{P}\left(s_{0}\right) \\
& =\kappa_{1} \dot{\tilde{d}}-\kappa_{3} \alpha_{2} s_{*}(0)+\Gamma_{1} \dot{s}_{0}+\Gamma_{2} \dot{P}\left(s_{0}\right)
\end{aligned}
$$

A double power reaching law given in [24] is used in order to improve the convergence rate of the system to the sliding mode surface $s_{1}=0$ in finite time:

$$
\dot{s}_{1}=-\lambda_{1} \operatorname{sig}\left(s_{1}\right)^{\gamma_{1}}-\lambda_{2} \operatorname{sig}\left(s_{1}\right)^{\gamma_{2}}
$$

where $\lambda_{1}>0, \lambda_{2}>0,0<\gamma_{1}<1, \gamma_{2}>1$.

Combining (14), (15) and Assumption 1, the differential equation of the disturbance observer is obtained:

$$
\begin{aligned}
\dot{\hat{d}}= & -\kappa_{2} \kappa_{3} \alpha^{2} s_{*}(0)+\kappa_{2}\left(\lambda_{1} \operatorname{sig}\left(s_{1}\right)^{\gamma_{1}}+\lambda_{2} \operatorname{sig}\left(s_{1}\right)^{\gamma_{2}}\right. \\
& \left.+\Gamma_{1} \dot{s}_{0}+\Gamma_{2} \dot{P}\left(s_{0}\right)\right)+(\bar{d}+\eta) \operatorname{sgn}\left(s_{1}\right)
\end{aligned}
$$

where $\eta>0$.

Theorem 1. If the sliding mode is as shown in (5) and (6) and the differential equation of the disturbance observer is as presented in (16), then the disturbance estimation error converges to 0 in the finite time $\tau=\tau_{1}+\tau_{2}$, where

$$
\begin{aligned}
\tau_{1} \leq & 2 V_{1}^{\left(1-\gamma_{1}\right) / 2}\left(s_{1}(0)\right) /\left(\phi\left(1-\gamma_{1}\right)\right) \\
\tau_{2} \leq & \left(\ln \left(V_{2}^{1 / 2}\left(s_{0}(0)\right)+\omega / \sqrt{2}\right)-\ln \right. \\
& (\omega / \sqrt{2})) / \Gamma_{1}
\end{aligned}
$$


Proof. A Lyapunov candidate function is chosen as

$$
V_{1}=1 / 2 s_{1}^{2}
$$

According to (14) and (16), the first order derivative of $s_{1}$ is presented as

$$
\begin{aligned}
\dot{s}_{1}= & \kappa_{1}(\dot{d}-\dot{\hat{d}})-\kappa_{3} \alpha^{2} s_{*}(0)+\Gamma_{1} \dot{s}_{0}+\Gamma_{2} \dot{P}\left(s_{0}\right) \\
= & \kappa_{1} \dot{d}-\kappa_{1}(\bar{d}+\eta) \operatorname{sgn}\left(s_{1}\right)-\lambda_{1} \operatorname{sig}\left(s_{1}\right)^{\gamma_{1}} \\
& -\lambda_{2} \operatorname{sig}\left(s_{1}\right)^{\gamma_{2}}
\end{aligned}
$$

Step 1: Considering (20) and the derivative of (19) yields

$$
\begin{aligned}
\dot{V}_{1} & =s_{1} \dot{s}_{1} \\
& =-\lambda_{1}\left|s_{1}\right|^{\gamma_{1}+1}-\lambda_{2}\left|s_{1}\right|^{\gamma_{2}+1}+\kappa_{1} s_{1} \dot{d}-\kappa_{1}(\bar{d}+\eta)\left|s_{1}\right| \\
& \leq-\lambda_{1}\left|s_{1}\right|^{\gamma_{1}+1} \\
& =-\lambda_{1} 2^{\left(\gamma_{1}+1\right) / 2} V_{1}^{\left(\gamma_{1}+1\right) / 2}
\end{aligned}
$$

Defining $\phi=\lambda_{1} 2^{\left(\gamma_{1}+1\right) / 2}$, then (22) is obtained as below:

$$
\dot{V}_{1}+\phi V_{1}^{\left(\gamma_{1}+1\right) / 2} \leq 0
$$

The solution of (22) is

$$
\tau_{1} \leq 2 V_{1}^{\left(1-\gamma_{1}\right) / 2}\left(s_{1}(0)\right) /\left(\phi\left(1-\gamma_{1}\right)\right)
$$

Hence $s_{1}$ will converge to zero in finite time. Then the terminal sliding mode function becomes

$$
\dot{s}_{0}=-\Gamma_{1} s_{0}-\Gamma_{2} P\left(s_{0}\right)
$$

Step 2: Choosing a Lyapunov candidate function as follows

$$
V_{2}=1 / 2 s_{0}^{2}
$$

Considering (24) and the derivative of (25) yields

$$
\begin{aligned}
\dot{V}_{2} & =s_{0} \dot{s}_{0} \\
& =-\Gamma_{1} s_{0}{ }^{2}-\Gamma_{2} s_{0} P\left(s_{0}\right)
\end{aligned}
$$

When $\left|s_{0}\right|=0$, then $\dot{V}_{2}=0$. When $\left|s_{0}\right| \neq 0$, an inequality can be acquired from (6) such that

$$
\left|P\left(s_{0}\right)\right| \geq \delta \varepsilon^{\theta}
$$

and the conclusion can be drawn from (6) that $s_{0}$ and $P\left(s_{0}\right)$ have the same positive or negative sign.

According to (27), $\dot{V}_{2}$ becomes

$$
\begin{aligned}
\dot{V}_{2} & =-2 \Gamma_{1} V_{2}-\Gamma_{2}\left|s_{0}\right|\left|P\left(s_{0}\right)\right| \\
& \leq-2 \Gamma_{1} V_{2}-\sqrt{2} \delta \varepsilon^{\theta} \Gamma_{2} V_{2}{ }^{1 / 2}
\end{aligned}
$$

The solution of (28) is

$$
\begin{aligned}
\tau_{2} \leq & \left(\ln \left(V_{2}^{1 / 2}\left(s_{0}(0)\right)+\omega / \sqrt{2}\right)-\ln \right. \\
& (\omega / \sqrt{2})) / \Gamma_{1}
\end{aligned}
$$

where $\omega=\delta \varepsilon^{\theta} \Gamma_{2} / \Gamma_{1}$.

It can be concluded from (9) that $\hat{d} \rightarrow d$ if and only if $s_{0} \rightarrow 0$ and $\dot{s}_{0} \rightarrow 0$, so the disturbance estimation error converges to 0 in finite time as required.

\section{Design of an Adaptive Sliding Mode Controller based on the Disturbance Observer}

The external disturbance can be estimated in finite time by the disturbance observer designed in Section 3. In this section, incorporating the disturbance observer presented above, an adaptive continuous sliding mode control method based on the disturbance observer is designed to suppress chattering, strengthen robustness and improve accuracy.

Combined with (3), a switching function is defined as below:

$$
S=e+C \int_{0}^{t} e(\mathrm{~T}) d T=0
$$

Considering the discontinuous reaching law with variable gain:

$$
\dot{S}=-K \operatorname{sgn}(S) / N(S)
$$

where $N(S)=\varpi+(1-\varpi) \exp \left(\ell|S|^{\partial}\right)<1, \ell>0$, $0<\varpi<1, \partial>0, K>0$.

An adaptive exponential reaching law is proposed by using the adaptive variable gain approach on the basis of (31).

$$
\dot{S}=-K_{1} S-\hat{K}_{2} \operatorname{sig}(S)^{\psi} / N(S)
$$

The adaptive reaching law is presented as

$$
\left.\dot{\hat{K}}_{2}=\gamma_{D} S \operatorname{sig}(S)^{\psi / N} / S\right)
$$

where $K_{1}>0, K_{2}>0, \hat{K}_{2}>0, \gamma_{D}>0,0<\psi<1$, and the definition of $N(S)$ is the same as (31).

The introduction of the adaptive law updates the system parameters to adapt to the variable states, to reduce chattering, reduce convergence time and compensate the control input.

Theorem 2. If the sliding mode is shown as (30), the reaching law is presented as (32), then taking into account (5) and (6), define the adaptive sliding mode controller for the CSTR as follows

$$
\begin{aligned}
& u=u_{1}+u_{2} \\
& u_{1}=-1 / \beta\left(f(x)+C e-\dot{x}_{2 d}\right) \\
& u_{2}=-1 / \beta\left(K_{1} S+\hat{K}_{2} \operatorname{sig}(S)^{\psi} / N(S)+\hat{d}\right)
\end{aligned}
$$

Then the sliding mode $S$ and the error $e$ are uniformly ultimately bounded.

Proof. A Lyapunov candidate function is chosen as

$$
V_{3}=1 /\left(2 S^{2}\right)+\tilde{K}_{2}^{2} /\left(2 \gamma_{D}\right)+V_{1}
$$

where $\tilde{K}_{2}=K_{2}-\hat{K}_{2}, \dot{\tilde{K}}_{2}=\dot{K}_{2}-\dot{\hat{K}}_{2}=-\dot{\hat{K}}_{2}$.

Differentiating (35) yields

$$
\dot{V}_{3}=S \dot{S}-\tilde{K}_{2} \dot{\hat{K}}_{2} / \gamma_{D}+\dot{V}_{1}
$$

Considering (34) and the derivative of (30) yields

$$
\begin{aligned}
\dot{S} & =\dot{e}+C e \\
& =\dot{x}_{2}-\dot{x}_{2 d}+C e \\
& =f(x)+\beta u+d-\dot{x}_{2 d}+C e \\
& =f(x)+\beta\left(u_{1}+u_{2}\right)+d-\dot{x}_{2 d}+C e \\
& =-K_{1} S-\hat{K}_{2} \operatorname{sig}(S)^{\psi} / N(S)+\tilde{d}
\end{aligned}
$$


The disturbance observer designed in Section 3 ensures that the disturbance estimation error converges to 0 in finite time, so the following assumption is imposed:

Assumption 3. The disturbance estimation error is bounded and satisfies

$$
|\tilde{d}| \leq K_{2}|S|_{\min }^{\psi} / N(S)
$$

where the definition of $K_{2}, \psi$ and $N(S)$ are as in (32).

It can be concluded that

$$
|\tilde{d}| \leq K_{2}|S|^{\psi} / N(S)
$$

Substitution of (33) and (37) into (36) yields

$$
\begin{aligned}
\dot{V}_{3}= & S\left(-K_{1} S-\hat{K}_{2} \operatorname{sig}(S)^{\psi} / N(S)+\tilde{d}\right) \\
& -\tilde{K}_{2} \dot{\hat{K}}_{2} / \gamma_{D}+\dot{V}_{1} \\
\leq & -K_{1} S^{2}-\hat{K}_{2} S \operatorname{sig}(S)^{\psi} / N(S)+|S||\tilde{d}| \\
& -\tilde{K}_{2} \dot{\hat{K}}_{2} / \gamma_{D}+\dot{V}_{1} \\
\leq & -K_{1} S^{2}-\hat{K}_{2}|S|^{\psi+1} / N(S)+K_{2}|S|^{\psi+1} \\
& / N(S)-\tilde{K}_{2} \dot{\hat{K}}_{2} / \gamma_{D}+\dot{V}_{1} \\
= & -K_{1} S^{2}+\tilde{K}_{2}|S|^{\psi+1} / N(S) \\
& -\tilde{K}_{2} \dot{\hat{K}}_{2} / \gamma_{D}+\dot{V}_{1} \\
= & -K_{1} S^{2}+\dot{V}_{1}<0
\end{aligned}
$$

Consequently, the sliding mode $S$ and the error $e$ are uniformly ultimately bounded.

\section{Simulation and Analysis}

Three algorithms are simulated in this section, and the corresponding control laws and parameters are presented as follows.

The first method is the adaptive sliding mode control based on disturbance observer (DOASMC) as presented in this paper. The control law has been given as (34) and the parameters are selected as $a=1.0, D a=0.072, \gamma=20.0$, $b=-8.0, \beta=0.3, c=20.0, \alpha=1.0, \Gamma_{1}=\Gamma_{2}=5.0, p=3$, $q=5, \varepsilon=0.001, \delta=0.5, \lambda_{1}=\lambda_{2}=6.0, \gamma_{1}=3 / 5, \gamma_{2}=5 / 3$, $\eta=0.001, C=1.0, \varpi=0.5, \ell=1.0, \partial=1.0, \gamma_{D}=1.0$, $K_{1}=10.0, \psi=3 / 5, d=0.01 \sin (\pi \tau)$. The initial values of $x_{1}$ and $x_{2}$ are 0.3 and 0.5 respectively, and the expected value of temperature is $x_{2 d}=1.0$.

For the second method (denoted as DOCRLSMC), the adaptive reaching law (32) is replaced with a constant reaching law, and the control law is shown as below:

$$
\begin{aligned}
& u=u_{1}+u_{2} \\
& u_{1}=-1 / \beta\left(f(x)+C e-\dot{x}_{2 d}\right) \\
& u_{2}=-1 / \beta(\eta \operatorname{sgn}(S)+\hat{d})
\end{aligned}
$$

where $\eta=1.0$, and other parameters take the same values as (34).

For the third method (denoted as SALASMC), the external disturbance is estimated with a simple adaptive law $(k S+\eta \operatorname{sgn}(S))$, and the control law is given as below:

$$
\begin{aligned}
& u=u_{1}+u_{2} \\
& u_{1}=-1 / \beta\left(f(x)+C e-\dot{x}_{2 d}\right) \\
& u_{2}=-1 / \beta\left(K_{1} S+\hat{K}_{2} \operatorname{sig}(S)^{\psi} / N(S)\right. \\
& +(k S+\eta \operatorname{sgn}(S)))
\end{aligned}
$$

where $k=3.0, \eta=1.0$, and other parameters take the same values as (34).

The simulation results are shown in the Fig.1-Fig.7.

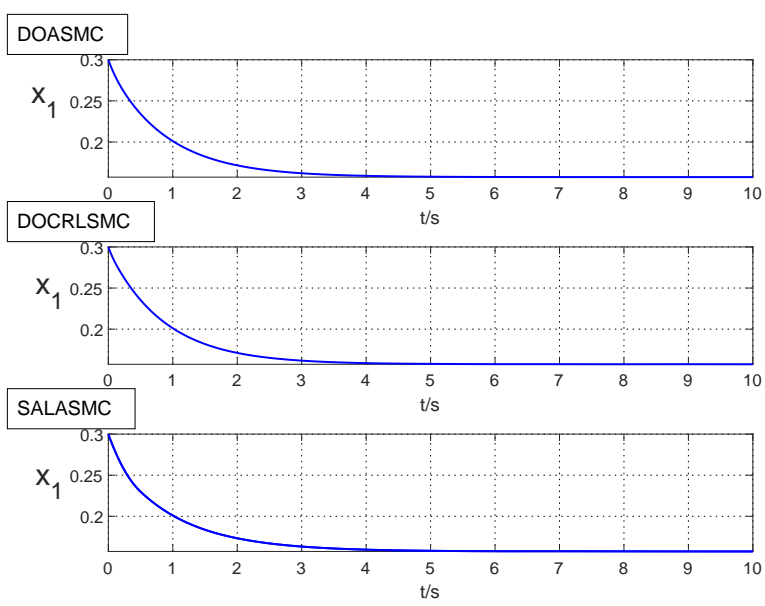

Fig. 1: Concentration response curves for the three methods

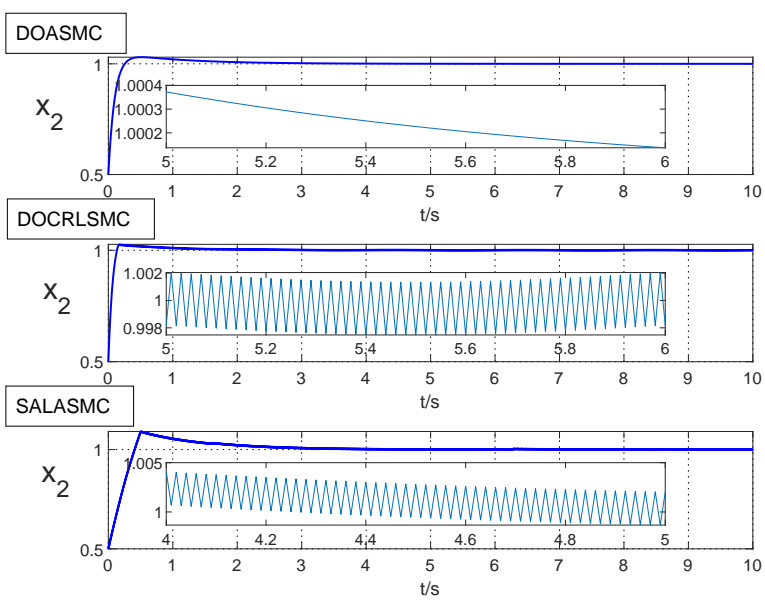

Fig. 2: Temperature response curves for the three methods

Fig.1-2 show that the concentration and temperature are gradually stabilized in finite time, and the states of Fig. 2 perform as expected. Fig. 3 indicates that the temperature errors converge to zero gradually. However, there is obvious chattering in the temperature response and temperature error response with DOCRLSMC. The settling time using the SALASMC is also extended.

Fig.4 illustrates that the states of the system approach the sliding mode surface $S=0$ at first, and after that, they converge asymptotically to zero along the sliding mode surface. It should be noted that he approach time of SALASMC is the longest. Besides, there is severe chattering in the second and the third curves in Fig.5, while the first one shows 


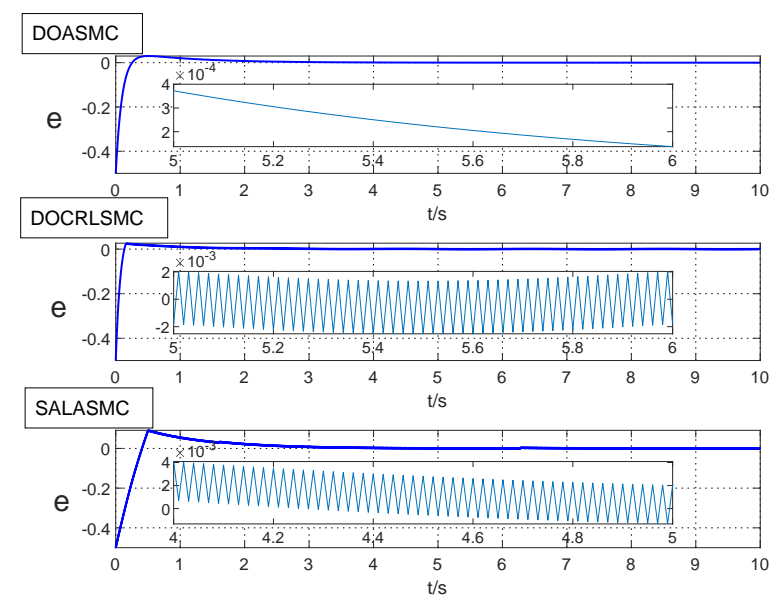

Fig. 3: Temperature error response curves for the three methods

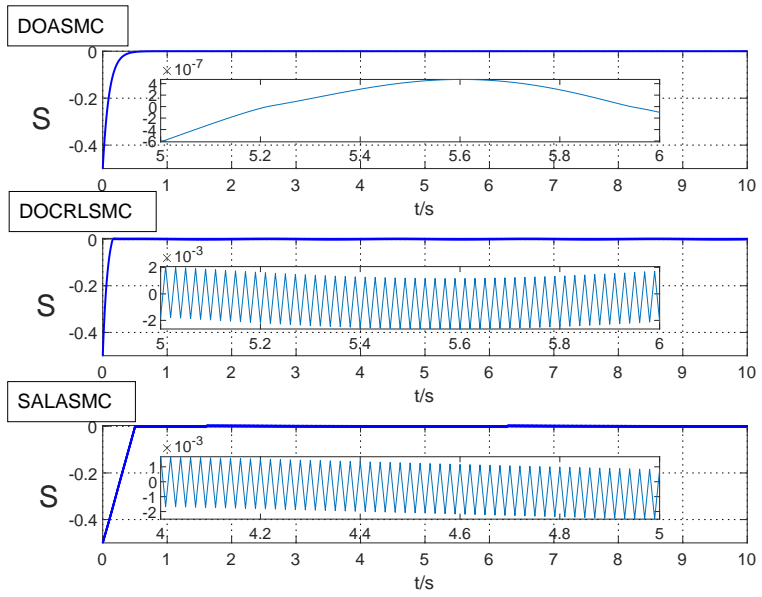

Fig. 4: Sliding mode curves for the three methods

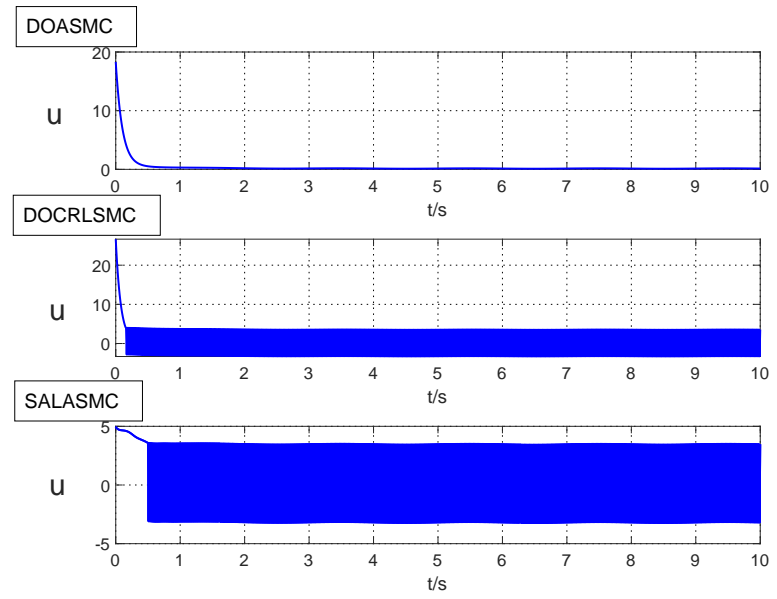

Fig. 5: Control input curves for the three methods

smooth control input. From the viewpoint of Fig.1 6, the first method performs best, because the disturbance observer can estimate the external disturbance and system uncertainty accurately in finite time, compensating the control gain, so that the chattering effects are reduced.

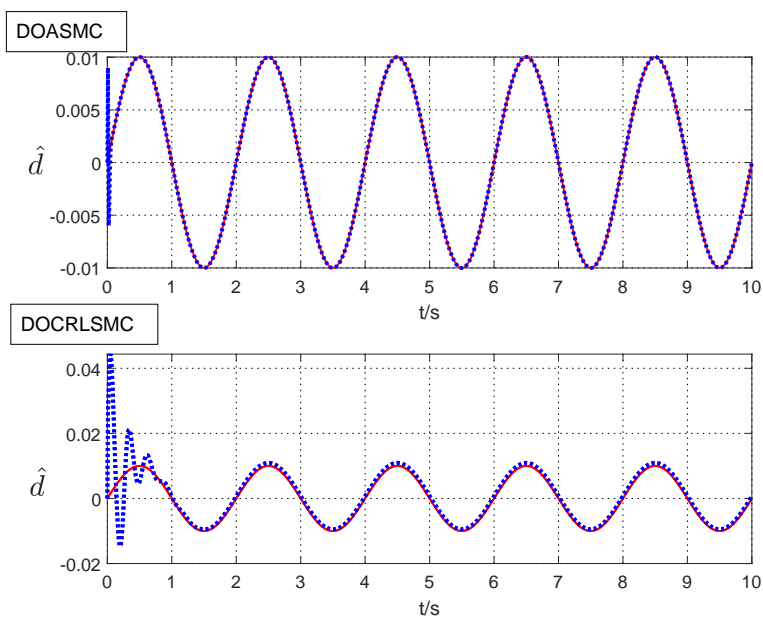

Fig. 6: Curves of disturbance estimation for the three methods

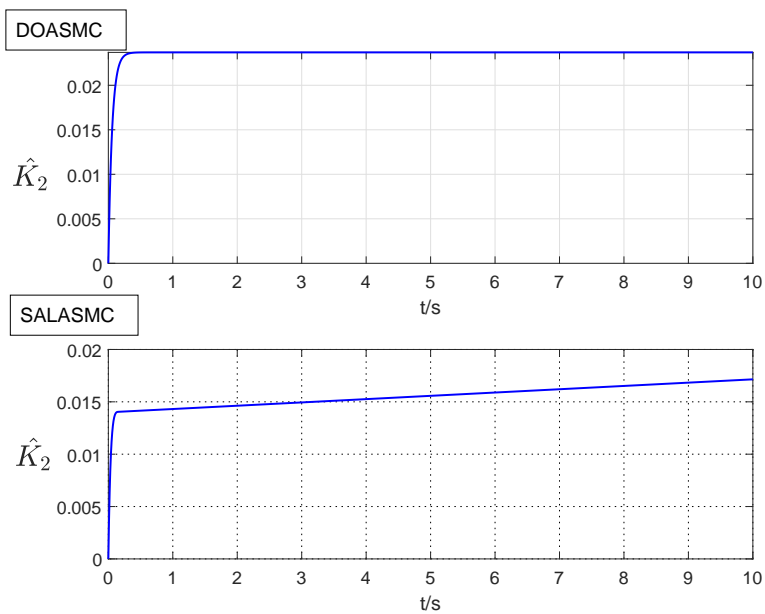

Fig. 7: The response curves of the adaptive parameters for the two adaptive methods

Fig. 7 shows the time response curves of the adaptive parameters. The system parameters are updated to adapt to the variable states by the adaptive laws. As is seen from Fig.1 5, the adaptive approach of SALASMC does not perform well.

In summary, the simulation results show that the proposed adaptive sliding mode control using a disturbance observer exhibits the best performance of the three methods considered.

\section{Conclusions}

In this paper, an adaptive sliding mode control incorporating a disturbance observer is presented to improve the control accuracy of a CSTR system. The disturbance observer can estimate the external disturbance and system uncertainty in finite time, compensate the control gain,= and better suppress chattering. The system parameters can be updated using the adaptive law, which improves the dynamic performance of the system and reduces the control input. Finally, the simulation results show that the proposed method can effectively estimate the external disturbance, reduce chattering and improve the control accuracy for the considered CSTR. 


\section{References}

[1] G. Feng. Overview of mechanically stirred reaction kettle, China Rubber/ Plastics Technology and Equipment (Plastics), 2015, 41(22): 56-57.

[2] N. Hoang, D. Dochain and B. Ydstie. Partial inventory control of the CSTR via reaction-dependent generalized inventories IFAC Proceedings Volumes, 2014, 47(3): 9123-9128.

[3] M. Bahita, K. Belarbi. Model reference neural-fuzzy adaptive control of the concentration in a chemical reactor (CSTR), 17th IFAC Conference on International Stability, Technology and Culture, 2016.

[4] S. Xie, Y. Xie, W. Gui, et al. Weighted-coupling CSTR modeling and model predictive control with parameter adaptive correction for the goethite process, Journal of Process Control, 2018, 68: 254-267.

[5] W. Chang. Nonlinear CSTR control system design using an artificial bee colony algorithm, Simulation Modelling Practice and Theory, 2013, 31: 1-9.

[6] S. Ge, C. Hang and T. Zhang. Nonlinear adaptive control using neural networks and its application to CSTR systems, Journal of Process Control, 1988, 9: 313-323.

[7] D. Gao, L. Huan and J. Cheng. Optimal output tracking control for chemical process of non-isothermal CSTR, 2016 Chinese Control and Decision Conference, 2016: 4588-4592.

[8] V. Ghaffari, S. Naghavi and A. Safavi. Robust model predictive control of a class of uncertain nonlinear systems with application to typical CSTR problems, Journal of Process Control, 2013, 23: 493-499.

[9] H. Mei, Y. Wang. Fast convergent sliding mode variable structure control of robot, Information and Control, 2009, 38(5): $552-557$.

[10] G. Huang. Terminal sliding mode control based on neural network disturbance observer, Journal of Jilin University: Engineering and Technology Edition, 2011, 41(6): 1726-1730.

[11] S. Singh, P. Srivastava, S. Janardhanan. Adaptive higher order sliding mode control for nonlinear uncertain systems, 5th IFAC Conference on Advances in Control and Optimization of Dynamical Systems, 2018.

[12] H. Ho, Y. Wong and A. Rad. Adaptive fuzzy sliding mode control with chattering elimination for nonlinear SISO systems, Simulation Modelling Practice and Theory, 2009, 17: 1199-1210.

[13] G. Bartolini, A. Levant, A. Pisano, et al. Adaptive secondorder sliding mode control with uncertainty compensation, International Journal of Control, 2016, 89(9): 1747-1758.

[14] H. Ozer, Y. Hacioglu and N. Yagiz. High order sliding mode control with estimation for vehicle active suspensions, Transactions of the Institute of Measurement and Control, 2017, 40(5): 1457-1470.

[15] S. Yu, X. Yu, B. Shirinzadeh, et al. Continuous fnite-time control for robotic manipulators with terminal sliding mode, Automatica, 2005, 41: 1957-1964.

[16] C. Hall, Y. Shtessel. Sliding mode disturbance observer based control for a reusable launch vehicle, Journal of Guidance, Control and Dynamics, 2006, 29(6): 1315-1328.

[17] X. Zeng, J. Wang, X. Wang, et al. Design of sliding mode controller based on SMDO and its application to missile control, Acta Aeronautica et Astronautica Sinica, 2011,35(5): 873-880.

[18] Q. Zhang, Q. Wu, C. Jiang, et al. Robust reconfi gurable tracking control of near space vehicle with actuator dynamic and input constraints, Control Theory and Applications, 2012, 29(10): 1263-1271.

[19] L. Yuan, F. Xiao, J. Shen, et al. Nonsingular terminal sliding mode control with disturbance observer for uncertain nonlinear systems, Control and Decision, 2014, 29(2): 353-357.
[20] F. Plestan, Y. Shtessel, V. Bregeault, et al. New methodologies for adaptive sliding mode control, International Journal of Control, 2010, 83(9): 1907-1919.

[21] Y. Shtessel, M. Taleb, F. Plestan. A novel adaptive-gain supertwisting sliding mode controller: methodology and application, Automatica, 2012(48): 759-769.

[22] C. J. Fallaha, M. Saad, H. Y. Kanaan, et al. Sliding-mode robot control with exponential reaching law, IEEE Transactions on Industrial Electronics, 2011, 58(2): 600-610.

[23] M. Colantonio, A. Desages, J. Romagnoli, et al. Nonlinear control of a CSTR: Disturbance rejection using sliding mode control, Process Design and Control, 1995, 34: 2383-2392.

[24] H. Zhang, J. Fan, F. Meng, et al. A new double power reaching law for sliding mode control, Control and Decision, 2013, 28(2): 289-293. 